EPJ Web of Conferences 66, 02106 (2014)

DOI: $10.1051 /$ epjconf/ 20146602106

(C) Owned by the authors, published by EDP Sciences, 2014

\title{
Recent shell-model results for exotic nuclei
}

\author{
Yutaka Utsuno ${ }^{1,2, a}$, Takaharu Otsuka ${ }^{2,3,4}$, Noritaka Shimizu ${ }^{2}$, Michio Honma $^{5}$, Takahiro Mizusaki ${ }^{6}$, \\ Yusuke Tsunoda ${ }^{3}$, and Takashi $\mathrm{Abe}^{3}$ \\ ${ }^{1}$ Advanced Science Research Center, Japan Atomic Energy Agency, Tokai, Ibaraki 319-1195, Japan \\ ${ }^{2}$ Center for Nuclear Study, University of Tokyo, Hongo, Bunkyo-ku, Tokyo 113-0033, Japan \\ ${ }^{3}$ Department of Physics, University of Tokyo, Hongo, Bunkyo-ku, Tokyo 113-0033, Japan \\ ${ }^{4}$ National Superconducting Cyclotron Laboratory and Department of Physics and Astronomy, Michigan State \\ University, East Lansing MI 48824, USA \\ ${ }^{5}$ Center for Mathematical Sciences, University of Aizu, Ikki-machi, Aizu-Wakamatsu, Fukushima 965-8580, \\ Japan \\ ${ }^{6}$ Institute for Natural Sciences, Senshu University, Tokyo, 101-8425, Japan
}

\begin{abstract}
We report on our recent advancement in the shell model and its applications to exotic nuclei, focusing on the shell evolution and large-scale calculations with the Monte Carlo shell model (MCSM). First, we test the validity of the monopole-based universal interaction $\left(V_{\mathrm{MU}}\right)$ as a shell-model interaction by performing large-scale shellmodel calculations in two different mass regions using effective interactions which partly comprise $V_{\mathrm{MU}}$. Those calculations are successful and provide a deeper insight into the shell evolution beyond the single-particle model, in particular showing that the evolution of the spin-orbit splitting due to the tensor force plays a decisive role in the structure of the neutron-rich $N \sim 28$ region and antimony isotopes. Next, we give a brief overview of recent developments in MCSM, and show that it is applicable to exotic nuclei that involve many valence orbits. As an example of its applications to exotic nuclei, shape coexistence in ${ }^{32} \mathrm{Mg}$ is examined.
\end{abstract}

\section{Introduction}

One of the major challenges for nuclear theory is to explain and predict the properties of all nuclei in a single framework. Exotic nuclei provide good testing grounds for probing its predictive power. Particularly important for this goal are determining the effective nuclear force unambiguously and establishing methodology of many-body problems which treats many-body correlations appropriately and accurately. This also applies to the shell model, one of the most successful nuclear-structure models in light to medium-heavy mass regions [1-4]. As for the former issue, although microscopic theories of effective interaction have been developed over decades, some empirical corrections are still needed for describing a wide range of nuclei belonging to a given shell. In particular, the monopole components of effective interactions - the parts responsible for the evolution of the shell structureare known to be subject to modification $[2,3,5]$. This so-called shell evolution plays a decisive role in the structure of exotic nuclei, and has been attracting much interest in this field. As for the latter

\footnotetext{
ae-mail: utsuno.yutaka@jaea.go.jp
} 
issue, while the shell model can treat many-body correlations straightforwardly by diagonalizing a many-body Hamiltonian matrix spanned by all the possible Slater determinants in a given valence shell, the dimension of the matrix immediately becomes too big to handle with the increase of the number of valence orbits and the number of valence particles [6].

In this paper, we report on recent advancement in shell-model description concerning exotic nuclei from the above points of view. Regarding the shell evolution, we are devoted to the effect of the tensor-force driven shell evolution of [7], which could be called Otsuka mechanism, on many-body properties such as the fragmentation of single-particle strength and the onset of deformation. It is shown that the monopole-based universal interaction $V_{\mathrm{MU}}$ [8], initially used to describe the evolution of spherical single-particle energies, is quite promising also as a shell-model interaction. With respect to solving many-body problems, we focus on recent advancement in the Monte Carlo shell model, which enables one to perform shell-model calculations beyond current computational limit $\left(\sim 10^{10}\right.$ in the $M$-scheme) in good accuracy.

\section{Shell evolution due to the effective interaction}

\subsection{Refined $V_{\mathrm{MU}}$ interaction}

In order to obtain a simple and universal effective interaction that describes the shell evolution, the monopole-based universal interaction $\left(V_{\mathrm{MU}}\right)$ has been proposed recently [8]. The original $V_{\mathrm{MU}}$ consists of a central force and a tensor force. Its tensor part is taken from the $\pi+\rho$ meson exchange force. The use of $\pi+\rho$ as the effective tensor force has a microscopic basis called "Renormalization Persistency" $[8,9]$ that the bare tensor force persists in the effective interaction. On the other hand, the central component is, in principle, much more complicated because of strong renormalization. A key idea of $V_{\mathrm{MU}}$ is that, at least as far as the monopole matrix elements are concerned, a Gaussian central force leads to a good approximation of the realistic effective interactions from which the $\pi+\rho$ tensor force is taken away [8].

Although the original $V_{\mathrm{MU}}$ is very simple but useful, there is room for refinement. One is to include the two-body spin-orbit force. In contrast to the central force and the tensor force, the spin-orbit force does not always cause a visible shell evolution, but sometimes plays a dominant role as shown later. In the present study, the spin-orbit part is taken from that of the M3Y interaction [10]. Another possible refinement is associated with the central part. A careful
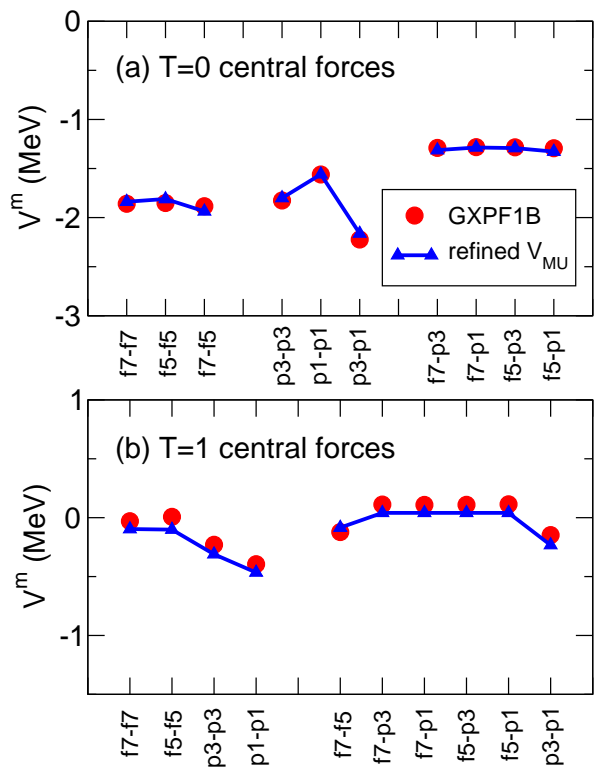

Figure 1. Comparison of the monopole matrix elements for the $p f$ shell between the central forces of GXPF1B and the refined $V_{\mathrm{MU}}$. comparison between the simple Gaussian force and the tensor-subtracted realistic force shows some disagreement about the strength of monopole interactions between higher nodal orbits such as those within $1 s_{1 / 2}$ (see Fig. 1 of [8]). We remedy this defect by introducing density dependence in the central force, following a simple recipe of Brown et al. [11]. The central force is here expressed as 
$V_{c}(r, R)=D(R) \sum_{S, T} f_{S, T} P_{S, T} \exp \left(-(r / \mu)^{2}\right)$ instead of Eq. (3) of [8]. The additional term $D(R)$, where $R=\left|\boldsymbol{r}_{1}+\boldsymbol{r}_{2}\right| / 2$, represents the density (or center-of-mass) dependence. In the same way as [11], $D(R)$ is assumed to take a form of $D(R)=1+A_{d} F(R)$, where $F(R)$ is proportional to the Woods-Saxon potential given by $F(R)=\left\{1+\exp \left(\left(R-R_{0}\right) / a\right)\right\}^{-1}$ with $R_{0}=1.2 A^{1 / 3} \mathrm{fm}$ and $a=0.6 \mathrm{fm}$. Here, $A$ is fixed to be 16 for simplicity. $D(R)$ behaves like $1+A_{d}$ at $R \sim 0$ and like 1 at $R \sim \infty$. The six parameters $f_{S, T}, \mu$ and $A_{d}$ are determined so as to obtain a good fit to the central force of the GXPF1B interaction [12] for the monopole part. The resulting values are $f_{0,0}=-140 \mathrm{MeV}, f_{1,0}=0$, $f_{0,1}=0.6 f_{0,0}, f_{1,1}=-0.6 f_{0,0}, \mu=1.2 \mathrm{fm}$, and $A_{d}=-0.4$, giving a good agreement with GXPF1B as shown in Fig. 1. This refined $V_{\mathrm{MU}}$ interaction is used as shell-model interactions which dominate the shell evolution and its related phenomena.

It is noted that, as discussed in [8], the shell evolution is affected by the central force and the tensor force in different ways. The tensor force strongly changes the spin-orbit splitting owing to the Otsuka mechanism [7]. On the other hand, the central force, hardly contributing to the modification of the spin-orbit splitting as presented in [8], works in such a way that the evolution depends on the node number instead. In this sense, the central force is responsible for the $\boldsymbol{l}^{2}$ term in terms of the Nilsson model.

\subsection{Neutron-rich $N \sim 28$ region}

The neutron-rich $N \sim 28$ region is a good testing ground for proving the shell evolution due to the tensor force because a direct test of the spin-orbit splitting can be carried out by comparing the distribution of spectroscopic strengths for one-proton removal reactions. Among them are very clear data for ${ }^{48} \mathrm{Ca}$ measured with the $\left(e, e^{\prime} p\right)$ reaction [13], carrying crucial information on the spin-orbit splitting for the proton $s d$ shell. The evolution of the proton $0 d$ spin-orbit splitting in going from ${ }^{40} \mathrm{Ca}$ to ${ }^{48} \mathrm{Ca}$ is dominated by the cross-shell interaction, $\left\langle s d 0 f_{7 / 2}|V| s d 0 f_{7 / 2}\right\rangle$ in particular. It is stressed that describing spectroscopic strengths is beyond single-particle models because they are in general highly fragmented.

Bearing this in mind, we have proposed a new shell-model interaction named SDPF-MU [14] to test our scheme described in Sect. 2.1 for constructing effective interactions. The cross-shell part is taken from the refined $V_{\mathrm{MU}}$ interaction. The intra-shell parts are based on USD [1] and GXPF1B [12], successful semi-empirical interactions for the $s d$ shell and the $p f$ shell, respectively. Since USD and GXPF1B are effective interactions for the $0 \hbar \omega$ space, SDPF-MU is designed for the model space consisting of pure $\hbar \omega$ configurations. While it is applicable to unnatural parity states by taking the model space composed of the $1 p-1 h$ states, we restrict ourselves in this paper to the $0 p-0 h$ states only.

Spectroscopic strengths for one-proton removal from ${ }^{48} \mathrm{Ca}$ are compared between the $\left(e, e^{\prime} p\right)$ experiment [13] and the shell-model calculation with SDPF-MU [14]. The calculation has excellently reproduced the experimental distribution of the spectroscopic strengths, and has presented that the distribution is quite sensitive to the tensor force. We have concluded in [14] that the proton $0 d$ spinorbit splitting is $\sim 2 \mathrm{MeV}$ reduced in going from ${ }^{40} \mathrm{Ca}$ to ${ }^{48} \mathrm{Ca}$ because of the tensor force and that the reduction is well described by the $V_{\mathrm{MU}}$ interaction.

The shell evolution due to the tensor force also changes nuclear deformation. A good example is found in neutron-rich silicon isotopes [14]. The $2_{1}^{+}$level in ${ }^{42} \mathrm{Si}$, measured at GANIL recently [15], is located very low, and thus indicates the disappearance of the $N=28$ magic number. The shell-model calculation with SDPF-MU well reproduces the evolution of the $2_{1}^{+}$levels in silicon isotopes. On the other hand, the $2_{1}^{+}$level in ${ }^{42} \mathrm{Si}$ is calculated to be too high when the tensor force is removed from the cross-shell interaction. The difference between the calculations with and without the tensor force 
is clearly seen in the potential energy surface (PES): the energy minimum is located at a stronglydeformed oblate shape and at a spherical shape for the former and the latter, respectively [14].

The strong deformation in ${ }^{42} \mathrm{Si}$ is induced by the quenching of shell gaps among the orbits belonging to the same major shell. This is known as the Jahn-Teller effect [16]. On the neutron side, the $N=28$ shell gap in silicon isotopes is reduced from the one in calcium isotopes because of the tensor force and the central force working cooperatively. The reduction of the $N=28$ shell gap alone, however, does not explain the origin of the strong deformation in ${ }^{42} \mathrm{Si}$ because deformation is less pronounced in ${ }^{44} \mathrm{~S}$ in spite of its narrower $N=28$ shell gap. Hence, the proton shell gap must be taken into consideration, too. As discussed in [14], silicon isotopes prefer an oblate deformation to a prolate deformation or a spherical shape when the $d_{5 / 2}$ orbit is close to $s_{1 / 2}$. The same is applied to neutron deformation for nuclei with $N=28$ involving $f_{7 / 2}$ and $p_{3 / 2}$. As a result, gaining much correlation energy, coherent oblate deformation between protons and neutrons occurs. This is a kind of the Jahn-Teller effect which is promoted by the shell evolution due to the tensor force, and is named "tensor-force-driven Jahn-Teller effect" [14]. While this strong mixing between a $j$ orbit and a $j-2$ one is in accordance with the quasi-SU(3) scheme [3, 17], the picture presented in [14] gives an intuitive explanation as to why $Z=14$ and $N=28$ prefer an oblate deformation to a prolate shape.

The two-body spin-orbit force can also be probed in the $s d-p f$ shell region as discussed by Sorlin an Porquet [18]. Comparing between ${ }^{36} \mathrm{~S}$ and ${ }^{34} \mathrm{Si}$, two additional protons in ${ }^{36} \mathrm{~S}$ predominantly occupy the $1 s_{1 / 2}$ orbit because both nuclei are nearly spherical. When $1 s_{1 / 2}$ is occupied, the tensor force has no effect. In addition, the spin-orbit splitting is hardly changed by the central force. On the other hand, the two-body spin-orbit force has relatively large monopole matrix elements for $1 s_{1 / 2}-1 p_{1 / 2}$ and $1 s_{1 / 2}-1 p_{3 / 2}$ which can be opposite in sign, thus playing a dominant role in the evolution of the $1 p$ spinorbit splitting. The low-lying levels in ${ }^{37} \mathrm{~S}$ and ${ }^{35} \mathrm{Si}$ calculated with SDPF-MU are shown in Fig. 2 together with their spectroscopic factors. For both nuclei, the level dominated by $1 p_{3 / 2}$ and the one dominated by $1 p_{1 / 2}$ are clearly seen, and the splitting between them is changed from $1.9 \mathrm{MeV}$ to $1.1 \mathrm{MeV}$ in going from ${ }^{37} \mathrm{~S}$ to ${ }^{35} \mathrm{Si}$. This result quantitatively agrees with a recent experiment [18], confirming the validity of the spin-orbit force in the M3Y interac-

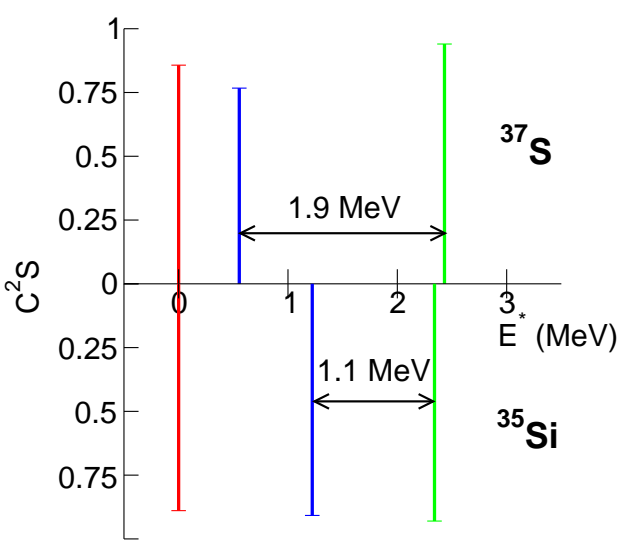

Figure 2. Low-lying energy levels and their spectroscopic factors obtained by one-neutron transfer reactions to ${ }^{37} \mathrm{~S}$ (upper) and ${ }^{35} \mathrm{Si}$ calculated with SDPF-MU. The red, blue and green lines stand for $0 f_{7 / 2}, 1 p_{3 / 2}$ and $1 p_{1 / 2}$, respectively. tion.

\subsection{Antimony isotopes}

With the proton number 51, antimony isotopes are expected to have one-proton single-particle states on top of the tin $(Z=50)$ core as low-lying states and thus to provide a good opportunity for probing the shell evolution. A recent experiment of the $\operatorname{Sn}(\alpha, t)$ reaction by Schiffer et al. has found that the spectroscopic strengths for the $7 / 2_{1}^{+}$and the $11 / 2^{-}$states are rather stable from $N=62$ to 74 and that they are probably close to unity [19]. The evolution of the $g_{7 / 2}-h_{11 / 2}$ splitting based on [19], indicating considerable reduction of the spin-orbit splitting for larger $N$, can only be accounted for by a mean-field model with the tensor force [20]. 
The single-particle character of low-lying levels in antimony isotopes is, however, a controversial issue. Sorlin and Porquet have claimed [21], on the basis of the $\left({ }^{3} \mathrm{He}, d\right)$ reactions leading to spectroscopic strength $\sim 0.5[22]$, that the $11 / 2^{-}$states are strongly correlated states rather than pure $h_{11 / 2}$ single-particle states, affected by states such as $\pi d_{5 / 2} \otimes v\left(3_{1}^{-}\right)$. Hence nuclear-structure calculations that can describe those correlated wave functions are needed to extract quantitative information on the shell evolution due to the effective interaction.

We have performed shell-model calculations for antimony isotopes in the full $1 d_{5 / 2}-0 g_{7 / 2}-0 h_{11 / 2^{-}}$ $2 s_{1 / 2}-1 d_{3 / 2}$ space. In order to obtain good tin-core states, we have adopted a semi-empirical neutronneutron interaction named SNBG3 [23] whose matrix elements are adjusted to fit experimental levels in tin isotopes including $33_{1}^{-}$states. The proton-neutron interaction, which is to be examined in this study, is taken from the refined $V_{\mathrm{MU}}$ interaction with one modification: the overall strength of the central force is multiplied by 0.84 so as to achieve a good fit to experimental one-proton separation energies along the antimony isotope chain. The proton single-particle energies are determined to reproduce experimental one-proton levels in ${ }^{133} \mathrm{Sb}$.

The calculated energy levels in antimony isotopes are compared with experimental data in Fig. 3, well reproducing the evolution of the energy levels. In order to see the interplay between the shell evolution and correlation, the effective single-particle energies (ESPE) [24] are also presented in Fig. 3. The ESPE1 is the usual ESPE: filling configurations are assumed. In the present case, sub-shell closures at $N=50,64$ and 82 are considered. Besides the ESPE1 which includes no correlation, neutron-neutron correlation can be taken into account in the ESPE by replacing the neutron filling wave function with the ground state of tin isotopes obtained by the shell-model calculation. The ESPE2 is defined in this manner. The ESPE1 follows the experimental $11 / 2_{1}^{-}$levels rather well as demonstrated already [7], but the $11 / 2_{1}^{-}$levels calculated with the ESPE2 are shifted $\sim 0.5 \mathrm{MeV}$ higher around the mid-shell isotopes. This is due to the fragmentation of the neutron occupation numbers, which makes the evolution of the levels milder. When protonneutron correlation is included in the full shellmodel calculation, $11 / 2_{1}^{-}$levels are pulled down again around the mid-shell isotopes, and are

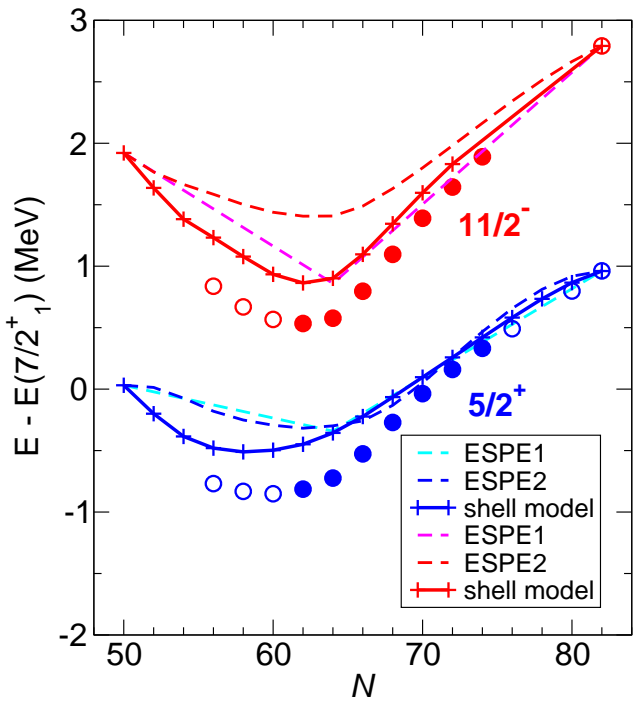

Figure 3. The $11 / 2_{1}^{-}$and $5 / 2_{1}^{+}$levels relative to $7 / 2_{1}^{+}$ in antimony isotopes compared between experiment (circles) and theory (lines). The filled and open circles are experimental levels whose spectroscopic factors have been measured and unmeasured, respectively. See the text for details. close to experimental data.

The spectroscopic factors for the $5 / 2_{1}^{+}$, $7 / 2_{1}^{+}$, and $11 / 2_{1}^{-}$states calculated with the shell model are reduced to $\sim 0.6$ for ${ }^{113-125} \mathrm{Sb}$, supporting the values extracted from the $\left({ }^{3} \mathrm{He}, d\right)$ reactions [22]. This sizable deviation from the single-particle limit accounts for the evolution of the magnetic moment from $N=82$ to lower neutron numbers. Nevertheless, the evolution of the $11 / 2_{1}^{-}-7 / 2_{1}^{+}$spacing is rather close to that of the naive effective 
single-particle energies because of the cancellation between neutron-neutron correlation energy and proton-neutron correlation energy.

\section{Advanced Monte Carlo shell model}

\subsection{Brief overview of recent development}

The Monte Carlo shell model (MCSM) has been proposed in 1995 in order to overcome the limit of the conventional exact-diagonalization method for performing shell-model calculations (see [25] for a review of its early development). The many-body wave function adopted by MCSM is usually expressed with a small number of selected deformed Slater determinants with symmetry restoration. When this number, called MCSM basis dimension $N_{b}$, increases, the energy of the MCSM wave function is converging typically at around $N_{b} \sim 100$ even if the total dimension is several orders larger. MCSM is thus characterized as importance truncation. Although MCSM has been successfully applied to several systems beyond exact diagonalization [25], application to huge model spaces consisting of many major shells was not feasible at that time [25] because of the limitation of realistic computational time.

Aiming at extending the applicability of MCSM, we have recently developed MCSM further (See [26] for a review of recent advancement). The computer code is completely renewed to be a hybrid parallel code using OpenMP and MPI in order to run on modern supercomputers such as the K computer [27]. Furthermore, a novel method for estimating the exact solution in good accuracy has been introduced [29]. The energy calculated with MCSM is converging for a sufficiently large $N_{b}$, but it is nevertheless difficult to find the exact energy from the plot of the energies as a function of $N_{b}$. The new method utilizes energy variance $\left\langle H^{2}\right\rangle-\langle H\rangle^{2}$, which vanishes for eigenstates, as a measure of proximity to the exact solution. More specifically, an energy plot is drawn as a function of the energy variance instead of $N_{b}$, and the plot is extrapolated to the point whose energy variance vanishes. See [28] for more details about the energy-variance extrapolation method. This method has been applied to MCSM and confirmed to work very well [29]. As another methodological advancement, the new MCSM method generates basis states more efficiently than the original method does. In the original method, candidates for a new basis state are generated in a stochastic way, and the one that gives the lowest energy is adopted among them. On the other hand, the new MCSM searches for a local minimum of energy by implementing the conjugate gradient method [30], thus reaching lower energy.

Large-scale calculations are facilitated also by efficient use of computational resources. At the level of single-core efficiency, it is desirable to reach as close as possible to theoretical peak performance. From this aspect, the new MCSM code implements an efficient method for computing the Hamiltonian overlap which is the most time-consuming part in the total calculation [31]. The high efficiency is attributed to transformation to a multiplication of dense matrices which requires much less memory access than arithmetic operations. The resulting performance typically attains $\sim 80 \%$ of the theoretical peak performance, which is a factor of 5-10 larger than that of the original MCSM. High parallel efficiency is also important. For this purpose, the ratio of computation to the amount of communication is enlarged in the new code: while the computation of one quantum-number-projected matrix element is divided by the number of cores in the original code, multiple matrix elements are treated at the same time in the new code. As a result, the parallel efficiency achieved by the new MCSM code is excellent even on a system with tens of thousands of CPU cores [26].

\subsection{Applications to exotic nuclei}

Owing to the advancement in MCSM described above and rapid increase of computing power, very large-scale shell-model calculations adopting multiple major shells are feasible now. Together with the 

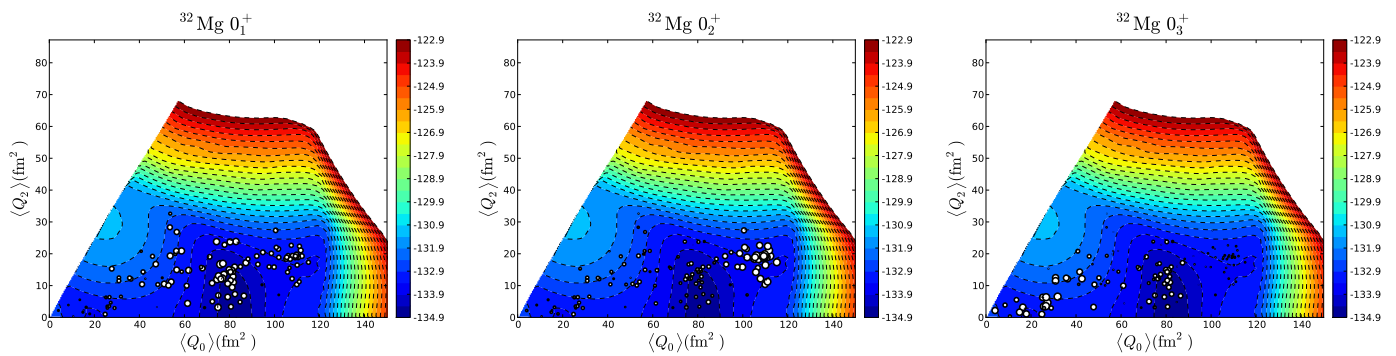

Figure 4. Distribution of the MCSM basis states for the $0_{1}^{+}$(left), $0_{2}^{+}$(center) and $0_{3}^{+}$(right) states in ${ }^{32} \mathrm{Mg}$ plotted on the potential energy surface. The position and the size (i.e., area) of each circle represent the deformation of each basis state and its overlap probability with the MCSM wave function, respectively.

ab initio shell model [32], exotic nuclei provide good opportunities for the advanced MCSM because a very large model space is often needed to examine the appearance and disappearance of magic numbers. Currently the most intensively and extensively studied is the neutron-rich chromium-nickel region, the results of which were reported in this conference [33]. Here, we show a preliminary result on the "island of inversion" region [34], in which the $N=20$ magic number is known to disappear. We now focus on the low-lying $0^{+}$states in ${ }^{32} \mathrm{Mg}$ because an anomalously low $0^{+}$state has been found recently [35]. Using an interaction for the full $s d-p f$ shell based on SDPF-MU [14], the lowest three $0^{+}$states are calculated with MCSM. In order to clarify the shape fluctuation of the wave functions obtained with MCSM, we adopt a visualization method introduced in [26]: for each MCSM wave function of interest, overlap probabilities with the basis states are calculated, and then the distribution of the overlap probabilities against intrinsic deformation is plotted as shown in Fig. 4 . While the ground state is dominated by the $2 p-2 h$ states with deformations around the energy minimum on the potential energy surface, the $0_{2}^{+}$and $0_{3}^{+}$states are dominated by the $4 p-4 h$ and $0 p-0 h$ states with larger and smaller deformations, respectively. It would be interesting to investigate whether those wave functions are able to explain the two-neutron transfer data [35].

\section{Summary}

Two important ingredients of nuclear-structure theory are effective interaction and many-body problems. In this paper, we survey our recent advancement in those issues in the framework of the shell model. Applied to the $N \sim 28$ neutron-rich region and antimony isotopes, the monopole-based universal interaction $V_{\mathrm{MU}}[8]$ is shown to be quite useful not only as an interaction that gives the shell evolution but also as a shell-model effective interaction. More specifically, we have demonstrated that the $\pi+\rho$ meson-exchange tensor force accounts for the evolution of the spin-orbit splitting by means of excellent agreement in the distribution of spectroscopic factors in ${ }^{48} \mathrm{Ca}$ and in the evolution of the energy levels in antimony isotopes. Those results support the universality of the effective tensor force suggested by microscopic effective-interaction theories [8,9]. We have also developed large-scale shell-model calculations with the Monte Carlo shell model (MCSM) in several aspects, achieving accurate estimate of the exact energy, high efficiency in the generation of basis states, and high computational performances in terms of single core and parallelism. Those developments are quite helpful in studying exotic nuclei involving many valence shells, as exemplified by the shape coexistence in ${ }^{32} \mathrm{Mg}$. 


\section{Acknowledgements}

This work was in part supported by JSPS KAKENHI Grant Numbers 21740204 and 23244049, and by the SPIRE Field 5, the MEXT of Japan. This work is a part of the CNS-RIKEN joint research project on large-scale nuclearstructure calculations.

\section{References}

[1] B. A. Brown and B. H. Wildenthal, Ann. Rev. Nucl. Part. Sci. 38, 29 (1988).

[2] M. Honma, T. Otsuka, B. A. Brown, and T. Mizusaki, Phys. Rev. C 69, 034335 (2004).

[3] E. Caurier et al, Rev. Mod. Phys. 77, 427 (2005).

[4] A. Holt, T. Engeland, M. Hjorth-Jensen, and E. Osnes, Nucl. Phys. A 634, 41 (1998).

[5] J. B. McGrory, B. H. Wildenthal, and E. C. Halbert, Phys. Rev. C 2, 186 (1970).

[6] G. F. Bertsch, D. J. Dean, and W. Nazarewicz, SciDAC Review 6, 42 (2007).

[7] T. Otsuka et al., Phys. Rev. Lett. 95, 232502 (2005).

[8] T. Otsuka et al., Phys. Rev. Lett. 104, 012501 (2010).

[9] N. Tsunoda, T. Otsuka, K. Tsukiyama, and M. Hjorth-Jensen, Phys. Rev. C 84, 044322 (2011).

[10] G. Bertsch, J. Borysowicz, H. McManus, and W. G. Love, Nucl. Phys. A 284, 399 (1977).

[11] B. A. Brown, W. A. Richter, R. E. Julies, and B. H. Wildenthal, Ann. Phys. 182, 191 (1988).

[12] M. Honma, T. Otsuka, and T. Mizusaki, RIKEN Accelerator Progress Report 41, 32 (2008).

[13] G. J. Kramer, H. P. Blok, and L. Lapikás, Nucl. Phys. A 679, 267 (2001).

[14] Y. Utsuno et al., Phys. Rev. C 86, 051031(R) (2012).

[15] B. Bastin et al., Phys. Rev. Lett. 99, 022503 (2007).

[16] H. A. Jahn and E. Teller, Proc. R. Soc. London, Ser. A 161, 220 (1937).

[17] A. P. Zuker, J. Retamosa, A. Poves and E. Caurier, Phys. Rev. C 52, R1741 (1995).

[18] O. Sorlin and M. -G. Porquet, Phys. Scr. T152, 014003 (2013).

[19] J. P. Schiffer et al., Phys. Rev. Lett. 92, 162501 (2004).

[20] T. Otsuka, T. Matuo and D. Abe, Phys. Rev. Lett. 97, 162501 (2006).

[21] O. Sorlin and M. -G. Porquet, Prog. Part. Nucl. Phys. 61, 602 (2008).

[22] M. Conjeaud et al., Nucl. Phys. A 117, 449 (1968).

[23] SNBG3 is a variant of SNBG1 of M. Honma et al. [RIKEN Accelerator Progress Report 45, 35

(2011) ], constructed to fit energy levels in tin isotopes including $3_{1}^{-}$states, too.

[24] Y. Utsuno, T. Otsuka, T. Mizusaki, and M. Honma, Phys. Rev. C 60, 054315 (1999).

[25] T. Otsuka et al., Prog. Part. Nucl. Phys. 47, 391 (2001).

[26] N. Shimizu et al., Prog. Theor. Exp. Phys. 2012, 01 A205 (2012).

[27] K Computer, http://www.aics.riken.jp/en/.

[28] T. Mizusaki and M. Imada, Phys. Rev. C 65, 064319 (2002); ibid. 67, 041301 (2003).

[29] N. Shimizu et al., Phys. Rev. C 82, 061305(R) (2010).

[30] N. Shimizu et al., Phys. Rev. C 85, 054301 (2012).

[31] Y. Utsuno, N. Shimizu, T. Otsuka, and T. Abe, Comput. Phys. Commun. 184, 102 (2013).

[32] T. Abe et al., Phys. Rev. C 86, 054301 (2012).

[33] Y. Tsunoda et al., contribution to this conference.

[34] E. K. Warburton, J. A. Becker, and B. A. Brown, Phys. Rev. C 41, 1147 (1990).

[35] K. Wimmer et al., Phys. Rev. Lett. 105, 252501 (2010). 